\title{
A Simple Method for High-Throughput Extract Prefractionation for Biological Screening
}

\author{
David R. Appleton, Antony D. Buss, and Mark S. Butler*
}

\begin{abstract}
The screening of crude natural product extracts can be problematic, especially in enzyme/protein-based assays, due to promiscuous inhibitors and interference compounds, while high salt and lipid content can significantly dilute compounds of interest. Described herein is a simple method for high-throughput fractionation of crude extracts that can help address some of these issues. Results obtained indicate a significant improvement in assay hit rates without a reduction of hit quality and show how prefractionation aids the discovery of compounds that would be undetected by screening crude extracts alone.
\end{abstract}

Keywords: Automation · High-throughput screening $\cdot$ Natural product · Prefractionation

\section{Introduction}

Natural products (NPs) have been a proven source of new drugs, ${ }^{[1]}$ with around $50 \%$ of marketed drugs derived from NPs ${ }^{[2]}$ and many NP-derived compounds undergoing clinical evaluation. ${ }^{[3]}$ However, despite this success, NP drug discovery has undergone many ups and downs in the last 20 years $^{[4]}$ due to alternative drug discovery methods that compete directly for resource. ${ }^{[5]}$ It was thought these alternative methods would offer quicker, more cost-effective routes to development, but this has not been the case with new drug launches falling to all time lows in recent years. ${ }^{[6]}$ This is especially the case in certain therapeutic areas; infectious disease, immunosuppression and oncology, where NPs have traditionally played an important role and continue to do so today. The main reason for the success of NPs in these therapeutic areas lies in the different chemical space occupied by NPs

\footnotetext{
${ }^{\star}$ Correspondence: Dr. M. S. Butler

MerLion Pharmaceuticals Pte Ltd

1 Science Park Road

The Capricorn \#05-01

Singapore Science Park II

Singapore 117528

Tel.: +65 68295600

Fax: +65 68295601

E-Mail: mark@merlionpharma.com
}

compared to most synthetics ${ }^{[7]}$ and intrinsic properties that enable some NPs to be orally active when they are predicted not to be. This was exemplified by Lipinski, who indicated in the 'Rule of Five' that oral bioavailability does not apply to NPs or to any molecule that is recognized by an active transport system. ${ }^{[8]}$ Another reason is that NPs are clearly produced by organisms for a purpose and biosynthetic genes have evolved specifically to produce compounds that interact with biological systems. ${ }^{[9]}$ For this reason, NPs should be expected to exhibit biological activity.

The advent of new technology has significantly decreased the time needed for bioassay-guided isolation and structure elucidation, allowing NP lead discovery to once again become a viable alternative for lead discovery. ${ }^{[5]}$ However, NPs should not be seen a panacea for all therapeutic targets, but used in conjunction with other methods for optimal results.

NP drug discovery must remain competitive through continual improvement in the hit identification, dereplication and isolation processes. Dereplication, or the identification of known compounds in active extracts, has improved markedly in recent years with the advent of more sensitive LCMS instruments and coupling to bioassay systems and NMR. [10] High-throughput screening (HTS) has allowed rapid screening of NP extracts, but also has introduced further problems due to the complexity of crude extracts and their interaction with some screening formats. Crude extracts are complex mixtures of compounds and, there- fore, offer more chemical diversity per assay point compared to most synthetic libraries. Unfortunately, the benefit of this is offset by the nature of some of the compounds commonly present in extracts. Promiscuous inhibitors and interference compounds are common in NP extracts and cause a significant number of false positives in HTS, while being time consuming to dereplicate early in the screening process. Some of these false positives are due to compounds that autofluoresce or absorb light at wavelengths that can interfere with assay readouts or are toxic in whole cell assays. Compounds that interact with screening targets in a non-specific manner also are common, such as detergent-like compounds and fatty acids/lipids. Recent publications have described how these compounds can form aggregates that interfere with certain types of assays. ${ }^{[11]}$ In addition, the presence of salts, lipids and media components in crude extracts dilute compounds of interest which reduces their chance of detection in assays. Advances in HTS assay formats and development of robust secondary assays have addressed some of these issues by aiding the removal of many false positives from screening hit lists. However, there is still the possibility of interesting compounds being missed due to low relative concentrations in an extract or masking by interference compounds. One way to address this is to fractionate the crude extracts before screening. Fractionation of extracts before screening can improve the chances of detecting interesting compounds that are present in minor amounts or whose activity is masked 
in the crude extract. The fractions are inherently less complex than crude extracts and allow compounds to be screened at higher concentrations, while separating interference compounds away from compounds of interest. Confinement of some classes of interference compounds into a single fraction also can allow identification of such issues early in a screening campaign. This can help make the dereplication process more efficient and direct compound identification efforts by providing options of selective screening or prioritisation of fractions. However, interfering/nuisance compounds that evade secondary assays will be identified more frequently since they are also enriched in fractions and screened at higher concentrations.

This paper describes a simple method for high-throughput prefractionation of crude NP extracts that has significantly increased hit rates and the ability to detect compounds of interest in screening programmes at MerLion Pharmaceuticals.

\section{Discussion}

Prefractionation, which is defined here as the fractionation of crude extracts before primary screening, can be undertaken using several methods. Prefractionation can result in few or many fractions, and these fractions can range from complex mixtures to semi-pure compounds. The simplest form of prefractionation is reduction of an extract to one fraction, such as the production of alkaloid-rich fractions using acid-base extraction protocols ${ }^{[12]}$ and the removal of larger molecular polyphenolic tannins from plant material by filtration through a solid absorbent such as polyamide.[13] Tannin removal provides plant extracts that are compatible with HTS, as these compounds interfere with many screening formats causing unmanageable hit rates. A simple prefractionation method using solid-phase extraction (SPE) cartridges was described for anionic polysaccharide removal and extract prioritisation of aqueous extracts with anti-HIV activity, ${ }^{[14]}$ while an automated SPE-based prefractionation method has been described by Thiericke and coworkers. ${ }^{[15]}$ Other methods used to produce prefractionated sample libraries include high-performance liquid chromatography (HPLC) ${ }^{[16]}$ and countercurrent chromatography $(\mathrm{CCC}) /$ centrifugal partition chromatography (CPC). ${ }^{[17]}$ Sequoia Sciences have developed a prefractionation procedure which is fully automated and integrates filtration, solid-phase extraction, automated flash chromatography and high-throughput parallel four-channel preparative HPLC. ${ }^{[18]}$ Some of these more complex prefractionation methods result in many fractions that are often mixtures of less than five com- pounds. In some cases, the active components can be identified directly from the prefractionated library samples using MS and NMR without requiring further isolation work.

The choice of prefractionation method depends upon cost considerations, required throughput and whether the fractions will be used for screening alone, dereplication or active compound identification as well. Our goal was to use the fractions for screening and to maintain a library of at least 120,000 fractions derived from methanol extracts of microbial cultures. After HTS hit identification, dereplication and isolation of active components could be achieved by using the remaining library sample and/or refermentation of the organism to give more extract. Given this goal, we needed to first choose an appropriate separation method. HPLC fractionation using reverse-phase $\mathrm{C}_{18}$ was chosen as it is easy to automate and separations could be performed on a scale large enough to provide material for several years of screening. Reverse-phase $\mathrm{C}_{18}$ would also provide reproducible and good compound resolution and recovery. The second key choice was the number of fractions to generate per sample. Drying of fractions would be the rate-limiting step in the process and too many fractions would make screening more costly and, in some cases, not feasible. In addition, when undertaking a NP HTS campaign, it is important that the maximum chemical diversity and, therefore, number of organisms are screened to provide the highest chance of success. Generation of large numbers of fractions per extract would limit the diversity screened significantly. However, too few fractions would result in losing some of the benefits of prefractionation, such as separation of interference compounds from interesting compounds and observation of activity trends. Clearly, a balance between the two extremes is required. It was decided that collection of four fractions starting at the end of the solvent front would provide enough compound resolution and concentration without being a significant burden to handle/screen. Since the solvent front contains mostly salts and accounts for the bulk of the extract mass there would be no advantage to collecting it into a discrete fraction so it was discarded. In addition, extremely late eluting, non-polar material was not collected as it contained mostly lipids of no interest, which would require a large volume of solvent to elute from the column and could interfere with assay readouts. Crude extracts were screened alongside the fractions to provide a control for the solvent front and lipophilic material, as well as for when activity is lost for other reasons during the prefractionation process.

Using a preparative HPLC fitted with a liquid handler and a short RP-C ${ }_{18}$ column
$(30 \times 19 \mathrm{~mm})$, a step gradient was developed that could fractionate up to $600 \mathrm{mg}$ of crude extract into four $13 \mathrm{ml}$ fractions in less than $7 \mathrm{~min}$. A step gradient was chosen to facilitate resolution of compounds into discrete fractions and improve the separation speed. The gradient was developed so that each fraction was contained in a single collection tube to allow for efficient drying and handling. After column washing and recycling, the overall cycle time was approximately $16 \mathrm{~min}$, therefore 48 samples could be fractionated in an overnight sequence. Fractions were dried during the following night using a Genevac HT-8 centrifugal evaporator. Once dry, the fractions were reformatted manually according to fraction number, re-dissolved in methanol and transferred to microtitre plates to form the mother screening plates. This set up allowed for the generation of up to 48,000 fractions per year and would provide enough material to last for 3-5 years of screening, resulting in a potential library size in excess of 120,000 fractions. Fractions were grouped into plates based on sample type and fraction number to allow for flexible screening choices. For example, it would be possible to select organism groups and certain fractions for screening if desired. This would be useful when the number of assay points available for a screen was limited.

Crude extracts were not weighed prior to prefractionation and the fractions generated were screened at concentrated equivalent dose to avoid time-consuming weighing steps. This approach was sufficient as other factors such as differences in salt and water content in extracts and varying solvation into methanol before HPLC would outweigh these approximations. In addition, accurate activity values were not required since the fractions were to be used only for primary screening. Crude extracts were prepared in the mother plates at $40 \mathrm{mg} / \mathrm{ml}$ in DMSO $(125 \mu \mathrm{l} /$ well $)$ while the fractions were prepared to the same volume to give an average concentration of approximately $10 \mathrm{mg} / \mathrm{ml}$, but represented a twelve times higher equivalent concentration than the crude extract, e.g. $25 \mathrm{mg}$ of crude extract is used to prepare five copies of the mother plate, whereas approximately $325 \mathrm{mg}$ of crude extract is fractionated to yield five copies.

\section{Results}

Examination of the chromatograms suggested that sufficient resolution of components could be achieved in less than 7 min with an extract mass of up to $600 \mathrm{mg}$ (Fig. 1). On average, $80-90 \%$ of the microbial extract mass comprised of insoluble material not injected and polar material that eluted with the solvent front. For a $500 \mathrm{mg}$ 
extract, fraction 1 weighed $20 \mathrm{mg}$ on average and fractions 2-4 weighed $6 \mathrm{mg}$.

Analysis of 1,700 active fractions from 11 screens confirmed that satisfactory resolution was achieved, with activity being concentrated into only one fraction in $84 \%$ of hits (Fig. 2). The activity distribution across the fractions also was reasonable (Fig. 3) but depended somewhat on the screen type. For example, for most whole cell screens the activity distribution was symmetrical around fractions 2 and 3, while for enzyme/protein based assays there was a significant skew towards fraction 4 , most likely due to assay interference. Interestingly, in $80 \%$ of the primary screening hits from prefractionated samples, the associated crude extract was not active, even though screened at four times the concentration of the fractions. In addition, fewer than $10 \%$ of the samples lost activity during the prefractionation process, validating the HPLC- $\mathrm{C}_{18}$ method. Examination of the screening results revealed that the activity of the fractions was enhanced by twelve times over the crude extracts on average. This concentration of activity led to a five to ten times increase in hit rate per extract due to prefractionation, easily accounting for the increase in assay points required to screen four fractions in addition to a crude extract. After screening prefractionated samples in 17 screens comprising of 400,000 assay points, $63 \%$ of active compounds isolated were derived from crude extracts that were inactive before prefractionation. These compounds would have been missed had only the crude extracts been screened.

One concern with prefractionation is the risk of concentrating weakly active or interference compounds and, therefore, lowering the quality of the hits. However, there was no reduction in mean activity values for isolated compounds or a significant increase in the proportion of interference compounds observed. The only issue was that hits from the prefractionated set required a longer time to isolate the active components. This was because in most cases compounds were present in low concentrations in the crude extract and therefore, the dereplication process required prior enrichment to detect known compounds and subsequent isolation work involved more steps.

Two examples from an antibacterial target demonstrate how prefractionation can result in compounds being discovered that would have been missed by screening only the crude extracts. In the first example the crude extract was not active whereas fraction 3 was active. Fig. 4 shows the dereplication data for the crude extract and active fraction. The plots are analytical HPLC coupled to bioassay graphs and demonstrate how there is no activity in the crude extract nor fractions at equivalent dose (Fig. 4a), but activity is revealed after prefractionation (Fig. 4b). The active compounds isolated

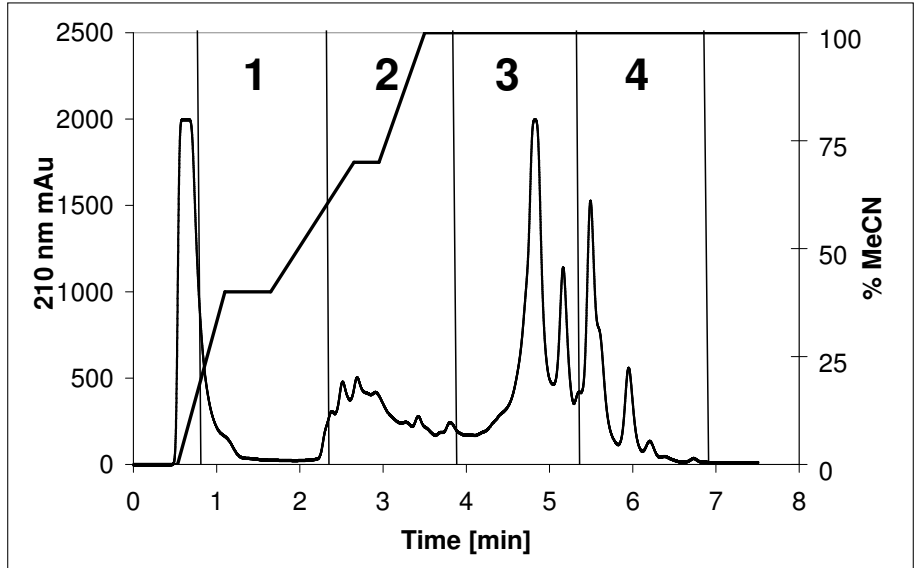

Fig. 1. HPLC chromatogram showing the gradient used and an example of the resolution achieved during prefractionation

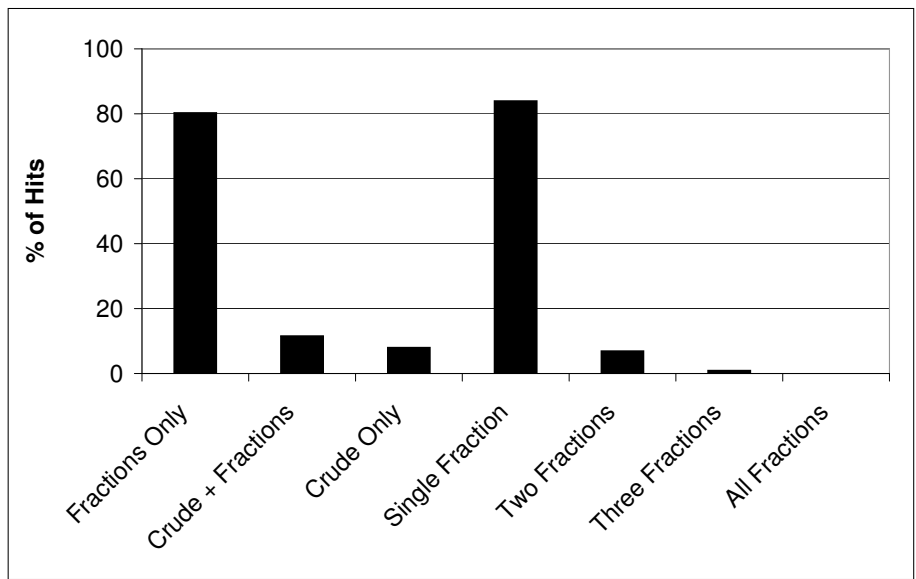

Fig. 2. Percentage make-up of HTS hits from eleven screens showing the activity enhancement and concentration due to prefractionation

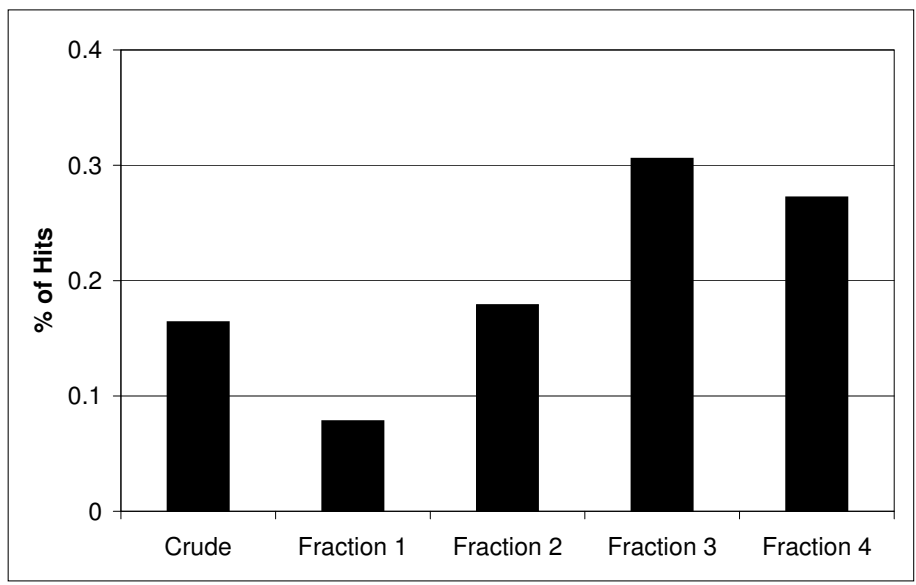

Fig. 3. Activity distribution between fractions of HTS hits from eleven screens.

from this sample were minor compounds $(0.3 \% \mathrm{wt} / \mathrm{wt})$ with mild activity $(30-50$ $\mu \mathrm{M})$, not potent enough to be detectable in the crude extract. In the second example again the crude extract was not active while fraction 3 was active, although the active compound exhibited good activity $(1 \mu \mathrm{M})$ and was present in relatively high concentration ( $1 \%$ extract weight). The dereplica- tion profile, in contrast to example 1 , shows that while no activity was observed in the crude extract, activity could be seen in the fractions when tested at equivalent dose (Fig. 5). Recombination of fractions and the solvent front traced the masking effect to the solvent front. Hence, this was a clear example of activity being unmasked by prefractionation. 


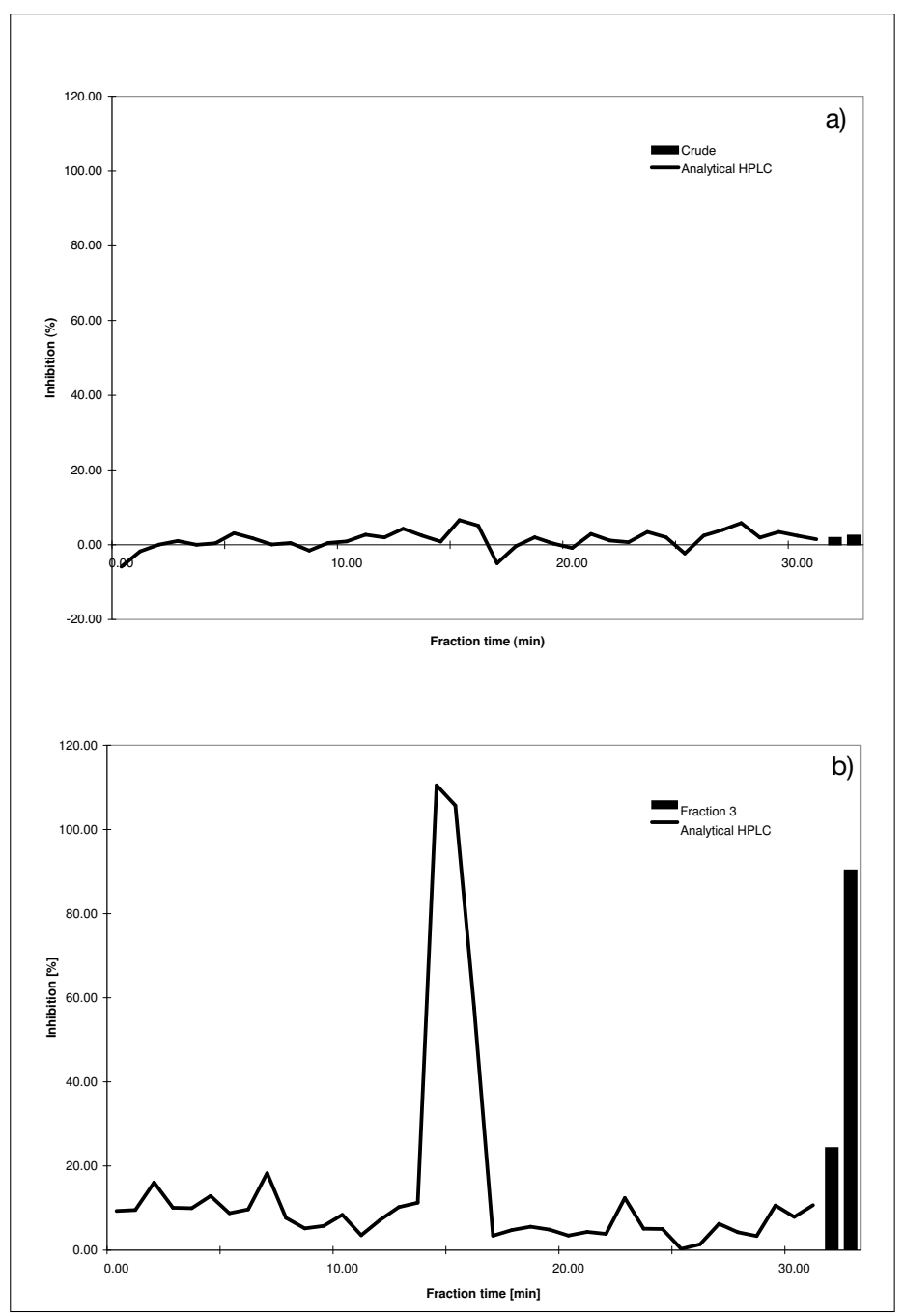

Fig. 4. Equivalent dose LC-bioassay plots of a sample demonstrating how minor compounds are concentrated by prefractionation by comparing a) inactive crude extract and b) active fraction

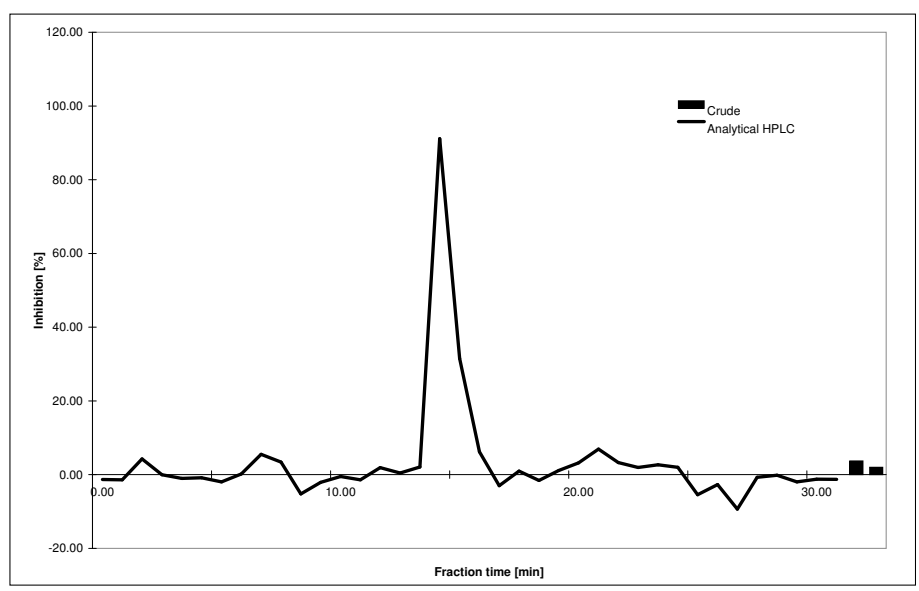

Fig. 5. Equivalent dose LC-bioassay plot demonstrating how a compound's activity can be un-masked by prefractionation.

\section{Experimental}

\section{Sample Preparation}

$\mathrm{MeOH}(1.4 \mathrm{ml})$ was added to approximately $500 \mathrm{mg}$ of the dried microbial extracts using a dispenser. The vials were capped, sonicated for $1 \mathrm{~h}$ and the solutions were transferred to a 96-well microtitre plate, which was then centrifuged.

\section{Preparative HPLC}

HPLC separation was performed on a Gilson 322/215 system equipped with dual wavelength detector using a Waters Xterra
RP-C ${ }_{18} 30 \times 19 \mathrm{~mm}$ column fitted with a $10 \mathrm{~mm}$ guard. Samples were injected onto the column $(1 \mathrm{ml})$ from the microtitre plate using the 215 liquid handler and fractions were collected into custom trays holding Genevac evaporator racks. The 215 liquid handler could accommodate 48 samples per sequence. A column wash was performed every 5-10 injections including injection of $\mathrm{CH}_{2} \mathrm{Cl}_{2}(2 \mathrm{ml})$ and DMSO at sequence end. A more thorough column back-washing was performed every 200-300 injections. Column life was typically 2000-2500 (guard 1000-1300) injections when the sample load was less than $500 \mathrm{mg}$ per injection, but decreased significantly when the loading was increased. Column condition was monitored by back pressure recording and regular injection of the following standards: adenosine, caffeine, 1,8-dihydroxyanthraquinone and albietic acid, all at $1 \mathrm{mg} / \mathrm{ml}$, were injected using the prefractionation protocol and monitored for peak shape, width and retention. Solvents used were Milli-Q H2O and HPLC grade MeCN (both containing $0.1 \%$ formic acid) and the flow rate during collection was $9 \mathrm{ml} / \mathrm{min}$. Fractions were collected every $1.44 \mathrm{~min}$ starting from the tail of the solvent front peak into $100 \times 16 \mathrm{~mm}$ tubes. The gradient used can be seen in Fig. 1.

\section{Solvent Removal}

Fractions were dried using a Genevac HT-8 centrifugal evaporator equipped with high power lamps and dry-pure. Initially, the set temperature was $40^{\circ} \mathrm{C}$ and pressure $40 \mathrm{mBar}$ for $45 \mathrm{~min}$ to remove most of the $\mathrm{MeCN}$. The pressure was then decreased to $6 \mathrm{mBar}$ for $9 \mathrm{~h} 30 \mathrm{~min}$ with direct heating to $40{ }^{\circ} \mathrm{C}$ for the first $30 \mathrm{~min}$. Finally, the pressure was further decreased to $2 \mathrm{mBar}$ for $3 \mathrm{~h}$ 30 min with direct heating to $35^{\circ} \mathrm{C}$ for the first $30 \mathrm{~min}$. Total drying time was therefore $13 \mathrm{~h} 45 \mathrm{~min}$ and the run was completed after a $2 \mathrm{~h}$ defrost. Maximum sample temperature during the drying was $35^{\circ} \mathrm{C}$.

\section{Assay Plate Preparation}

Once dry, fraction tubes were manually transferred into $10 \times 8$ format racks according to fraction number to allow for selective screening of fractions later. $\mathrm{MeOH}$ was added using a Tecan Genesis RSP 100 liquid handler and after sonication, dispensed into five copies of 96-well microtitre plates to form the assay mother plates.

\section{Acknowledgments}

We thank Nilar for initial work in development of the HPLC methods and Soon Geok Hong for her input into management of the prefractionation programme along with the many other participants at MerLion Pharmaceuticals. 
$\overline{[1]}$ a) W . Sneader, 'Drug Discovery: A History', John Wiley \& Sons, Chichester, 2005; b) D. J. Newman, G. M. Cragg, K. M. Snader, Nat. Prod. Rep. 2000, 17, 215; c) J. Drews, Science 2000, 287, 1960.

[2] D. J. Newman, G. M. Cragg, J. Nat. Prod. 2007, 70, 461 .

[3] a) M. S. Butler, Nat. Prod. Rep. 2005, 22, 162; b) Y.-W. Chin, M. J. Balunas, H. B. Chai, A. D. Kinghorn, AAPS J. 2006, 8, E239.

[4] a) R. M. Wilson, S. J. Danishefsky, J. Org. Chem. 2006, 71, 8329; b) I. Paterson, E. A. Anderson, Science 2005, 310, 451; c) A. M. Piggott, P. Karuso, Comb. Chem. High Throughput Screen. 2004, 7, 607; d) J. Y. Ortholand, A. Ganesan, Curr. Opin. Chem. Biol. 2004, 8, 271; e) H. G. Wildman, Fungal Diversity 2003, 13, 221; f) W. R. Strohl, Drug Discov. Today 2000, 5, 39; g) A. L. Harvey, Trends Pharmacol. Sci. 1999, 20, 196; h) L. J. Nisbet, M. Moore, Curr. Opin. Biotechnol. 1997, 8, 708.

[5] a) M. S. Butler, J. Nat. Prod. 2004, 67, 2141; b) F. E. Koehn, G. T. Carter, Nat. Rev. Drug Discov. 2005, 4, 206; c) G. G. Harrigan, G. H. Goetz, Comb. Chem. High Throughput Screen. 2005, 8, 529; d) V. P. Gullo, J. McAlpine, K. S. Lam, D. Baker, F. Petersen, J. Ind. Microbiol. Biotechnol. 2006, 33, 523.

[6] J. Owens, Nat. Rev. Drug Discov. 2007, 6, 99.

[7] a) T. Henkel, R. M. Brunne, H. Müller, F. Reichel, Angew. Chem., Int. Ed. 1999, 38 , 643; b) M. Feher, J. M. Schmidt, J. Chem. Inf. Comput. Sci. 2003, 43, 218; c) M. A. Koch, A. Schuffenhauer, M. Scheck, S. Wetzel, M. Casaulta, A. Odermatt, P. Ertl, H. Waldmann, Proc. Natl. Acad. Sci. USA 2005, 102, 17272.

[8] a) C. A. Lipinski, Drug Discov. Today: Technologies 2004, 1, 337; b) T. H. Keller, A. Pichota, Z. Yin, Curr. Opin. Chem. Biol. 2006, 10, 357; c) C. A. Lipinski, F. Lombardo, B. W. Dominy, P. J. Feeney, Adv. Drug. Deliv. Rev. 2001, 46, 3.

[9] a) D. H. Williams, M. J. Stone, P. R. Hauck, S. K. Rahman, J. Nat. Prod. 1989, 52, 1189; b) R. D. Firn, C. G. Jones, Nat. Prod. Rep. 2003, 20, 382.

[10] a) G. A. Cordell, C. W. W. Beecher, A. D. Kinghorn, J. M. Pezzuto, H. L. Constant, H. B. Chai, L. Fang, E.-K. Seo, L. Long, B. Cui, K. Slowing-Barillas, in 'Studies in Natural Products Chemistry: Bioactive Natural Products, Vol. 19, Structure and Chemistry (Part E)', Ed. Atta-ur-Rahman, Elsevier, Amsterdam, 1997, p. 749; b) T. O. Larsen, J. Smedsgaard, K. F. Nielsen, M. E. Hansen, J. C. Frisvad, Nat. Prod. Rep. 2005, 22, 672; c) R. T. Williamson, E. L. Chapin, A. W. Carr, J. R. Gilbert, P. R. Graupner, P. Lewer, P. McKamey, J. R. Carney, W. H. Gerwick, Org. Lett. 2000, 2 , 289; d) J. L. Wolfender, E. F. Queiroz, K. Hostettmann, Magn. Reson. Chem. 2005, 43, 697; e) K. Böröczky, H. Laatsch, I.
Wagner-Döbler, K. Stritzke, S. Schulz, Chem. Biodivers. 2006, 3, 622.

[11] a) K. E. Coan, B. K. Shoichet, Mol. Biosyst. 2007, 3, 208; b) B. K. Shoichet, Drug Discov. Today 2006, 11, 607.

[12] a) G. L. Silva, I.-K. Lee, A. D. Kinghorn, in 'Natural Product Isolation; Methods in Biotechnology, Vol. 4', Ed. R. J. P. Cannell, Humana Press, Totowa, 1998, p. 343. b) G. A. Cordell, M. L. Quinn-Beattie, N. R. Farnsworth, Phytother. Res. 2001, 15, 183.

[13] N. VanMiddlesworth, R. J. P. Cannell, in 'Natural Product Isolation; Methods in Biotechnology, Vol. 4', Ed. R. J. P. Cannell, Humana Press, Totowa, 1998, p. 279.

[14] J. H. Cardellina II, M. H. G. Munro, R. W. Fuller, K. P. Manfredi, T. C. McKee, M. Tischler, H. R. Bokesch, K. R. Gustafson, J. A. Beutler, M. R. Boyd, J. Nat. Prod. 1993, 56, 1123.

[15] I. Schmid, I. Sattler, S. Grabley, R. Thiericke, J. Biomol. Screening 1999, 4, 15.

[16] a) M. Stewart, R. J. Nash, M. I. ChicarelliRobinson, in 'Saponins in Food, Feedstuffs and Medicinal Plants' Eds. W. Oleszek, A. Marston, Kluwer Academic Publishers: Boston, 2000, p. 73; b) K. U. Bindseil, J. Jakupovic, D. Wolf, J. Lavayre, J. Leboul, D. van der Pyl, Drug Discov. Today 2001, 6, 840; c) U. Abel, C. Koch, M. Speitling, F.G. Hansske, Curr. Opin. Chem. Biol. 2002, 6, 453; d) Q. Jia, in 'Studies in Natural Products Chemistry: Bioactive Natural Products (Part J)', Ed. Atta-ur-Rahman, Elsevier, Amsterdam, 2003, p. 643; e) C. Koch, T. Neumann, R. Thiericke, S. Grabley, in 'Drug Discovery from Nature', Eds. S. Grabley, R. Thiericke, Springer, Berlin, 2000, p. 51.

[17] a) K. A. Alvi, in 'Biologically Active Natural Products: Pharmaceuticals', Eds. S. J. Cutler, H. G. Cutler, CRC Press, New York, 2000, p. 185; b) K. A. Alvi, J. Peterson, B. Hofmann, J. Ind. Microbiol. 1995, 15, 80; c) J. A. Armbruster, R. P. Borris, Q. Jiminez, N. Zamora, G. Tamayo-Castillo, G. H. Harris, J. Liq. Chromatogr. Relat. Technol. 2001, 24, 1827; d) K. Ingkaninan, A. Hazekamp, A. C. Hoek, S. Balconi, R. Verpoorte, J. Liq. Chromatogr. Relat. Technol. 2000, 23, 2195.

[18] a) G. R. Eldridge, H. C. Vervoort, C. M. Lee, P. A. Cremin, C. T. Williams, S. M. Hart, M. G. Goering, M. O'Neill-Johnson, L. Zeng, Anal. Chem. 2002, 74, 3963; b) J. F. Hu, E. Garo, G. W. Hough, M. G. Goering, M. O’Neil-Johnson, G. R. Eldridge. J. Nat. Prod. 2006, 69, 585; c) J. F. Hu, E. Garo, H. D. Yoo, P. A. Cremin, M. G. Goering, M. O'Neil-Johnson, G. R. Eldridge, Phytochemistry 2005, 66, 1077. 